\title{
An artificial intelligence strategy to detect damage from response measurements: application on an ancient tower
}

\author{
Gabriele Marrongelli ${ }^{1, *}$, Rafaelle Finotti ${ }^{2}$, Carmelo Gentile ${ }^{1}$, and Flavio Barbosa ${ }^{2}$ \\ ${ }^{1} \mathrm{ABC}$ Department, Politecnico di Milano, Milan, Italy \\ ${ }^{2}$ Department of Applied Computational Mechanics, Juiz de Fora, Brazil
}

\begin{abstract}
Automated modal identification procedures are attracting the interest of the Structural Health Monitoring (SHM) community as those techniques are capable of continuously providing information which are useful to timely assess the health state of a structure. Within this context, the paper presents the development and application of a vibration-based novelty detection strategy using automatically identified resonant frequencies and the Support Vector Machine (SVM) approach. The SVM is a popular technique for forming decision boundaries that separate data into two or more classes without any prior assumptions on the propriety of the data. The developed procedure is exemplified using frequency data collected during the continuous dynamic monitoring of a historic masonry tower that underwent slight permanent variation of the natural frequencies after the occurrence of a far-field earthquake. The obtained results highlight the capability of the novelty strategy to reveal slight damage and to detect anomalies in the structural behaviour.
\end{abstract}

\section{Introduction}

Among the different available approaches for Structural Health Monitoring (SHM), one of the most popular involves long-term vibration monitoring and the automated modal parameters estimation [1-4] from the responses continuously acquired in operational conditions. Nevertheless, the use of automated operational modal analysis (OMA) does not solve the problem of anomaly or damage detection by itself because the sensitive features (such as the resonant frequencies) exhibit fluctuations, strictly depending on the environmental and operational conditions, even larger than the ones determined by the structural changes [5]. Consequently, if the dynamic characteristics (or responses) of a structure are used for structural condition assessment, all variations due to anything other than structural changes have to be understood and accounted for. The occurrence of any changes is often detected through: (a) the application of appropriate statistical tools to remove the effects of changing environmental/operational conditions and (after an initial period needed to build and train the statistical models characterizing the normal conditions) to detect the possible novelty associated to an abnormal structural change (see e.g. [6-7]);

\footnotetext{
${ }^{*}$ Corresponding author: mailto:gabriele.marrongelli@polimi.it
} 
(b) the analysis of anomalies in the time evolution of natural frequencies, using unsupervised learning techniques [8-9]. It should be noticed that the proposed goal of the latter methods is to separate data characterizing different structural conditions, so that the occurrence of a structural change is detected.

This paper presents an efficient strategy of novelty detection using the frequency data as inputs for an artificial intelligence system based on a Support Vector Machine (SVM) algorithm. The reliability of the proposed approach is demonstrated using real data collected by a simple monitoring system installed on an ancient masonry tower in Mantua, Italy [10-11]. During the monitoring period examined in this paper, the tower underwent a far-field seismic event and a slight permanent variation in the natural frequencies of the tower has been observed after the seismic event. The SVM algorithm was firstly applied by using only the natural frequencies as inputs; furthermore, in order to evaluate the occurrence of false alarms, both the automatically identified frequencies and the measured temperature data were used as input parameters [12].

\section{Support Vector Machine}

SVM algorithms are aimed at separating two different classes using a discrimination function, which is computed within the classification of training datasets. Within the context of SHM and damage detection of civil engineering structures [9], two classes of data are assumed over time, corresponding to undamaged and damaged conditions: if the algorithm fails to distinguish both classes, it means that data belong to the same class and, consequently there is no structural damage. On the contrary, the successful detection of two classes implies that damage occurred. Although SVM algorithms are normally used for two-class classification problems, extension to multi-classes classification can be done.

The SVM is generally based on a geometric approach, consisting of the construction of an optimal separating surface - a hyper-plane - which divides the data population in two groups with different statistical characteristics. The hyper-plane is equidistant from the two classes, defining a margin zone between them. Similarly to Neural Network classification, the input data are supposed to belong to different classes and the outputs consist of the target binary vectors (labels) corresponding to each class. In case of linear separable data, the SVM algorithm searches the optimal solution to classify the data by maximizing the distance between the hyper-plane and the extreme values of the two classes (so called Support Vector). Several kernel functions can be used to make an appropriate construction of the hyper-plane. In this work, the Gaussian Radial Basis (GRB) function was adopted.

\section{Application to real data}

\subsection{Description of the monitored historic tower}

The Gabbia tower [10-11] (Fig. 1), with its $54.0 \mathrm{~m}$ height, is the tallest tower in Mantua. The tower was built in 1227 for defensive purposes by the Bonacolsi family, governing Mantua in the 13th century. The structure is built in solid brick masonry and the loadbearing walls are about $2.4 \mathrm{~m}$ thick, except in the upper levels, where the walls thickness decreases to about $0.7 \mathrm{~m}$. As shown in Fig. 1, the tower is nowadays part of an important palace, whose load-bearing walls seem to be not effectively connected to the tower; nevertheless, the several vaults and floors of the palace are directly supported by the tower. While the main part of the building - below the height of about $46.0 \mathrm{~m}$ from the ground level - does not exhibit any evident structural damage (with the materials being only affected by superficial decay), the upper part of the tower is in a poor state of preservation. 
(a)

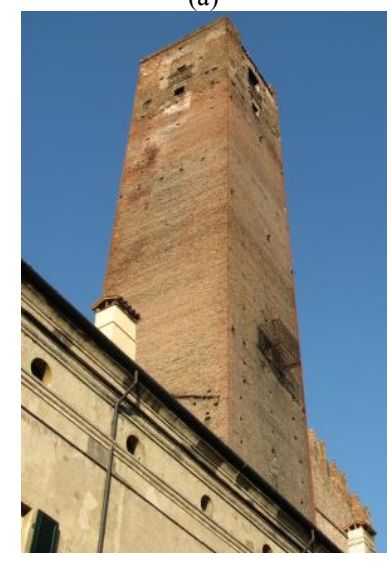

(b)

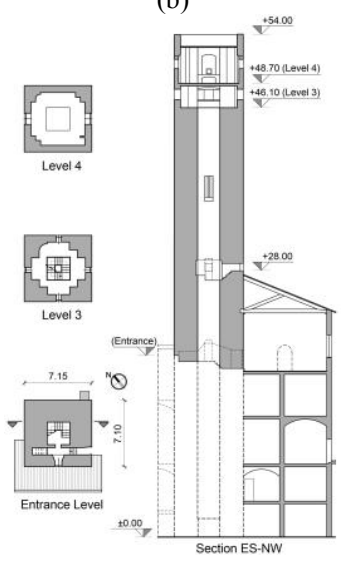

(c)

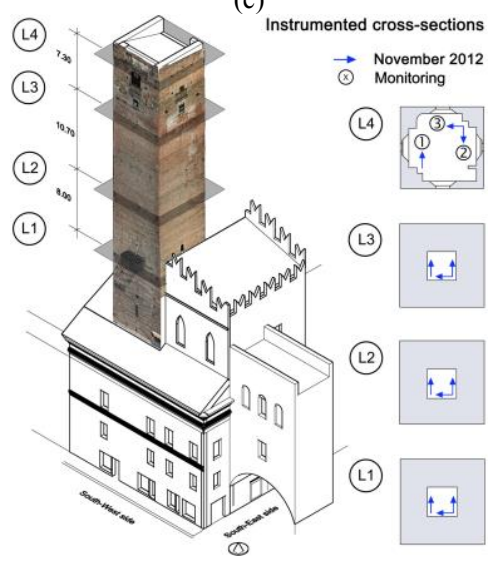

Fig. 1. (a) View of the Gabbia tower in Mantua, Italy; (b) Sections of the tower (dimensions in $\mathrm{m}$ ); (c) Instrumented cross-sections and layout of the accelerometers during the preliminary tests (November 2012) and the continuous dynamic monitoring.

In more details, at a distance of about $8.0 \mathrm{~m}$ from the top, the brick surface workmanship changes and the masonry quality significantly decreases (as it was confirmed by pulse sonic tests); furthermore, the presence of several structural discontinuities and the lack of mechanical connection between subsequent addings determined concerns about the seismic behaviour of the upper part of the tower.

\subsection{Dynamic characteristics of the tower and data analysis}

After preliminary ambient vibration tests (November 2012, Fig. 1c), a simple dynamic monitoring system was installed in the tower on December 17th, 2012 (and removed at the beginning of July 2015). The monitoring system (Fig. 1c) was composed of three piezoelectric accelerometers $(10 \mathrm{~V} / \mathrm{g}$ sensitivity), mounted on the cross-section at the crowning level of the tower, one temperature sensor, one 4-channels data acquisition system (24-bit resolution, $102 \mathrm{~dB}$ dynamic range and anti-aliasing filters) and one industrial $\mathrm{PC}$ on site, for the purpose of system management and data storage. A binary file, containing 3 acceleration time series and the temperature data, is created every hour, stored in the local PC and transmitted to Politecnico di Milano for signal processing.

The data recorded by the monitoring system were managed by a LabVIEW toolkit, including the (on-line or offline) execution of the following tasks: (i) creation of a database with the original data in compact format; (ii) data pre-processing; (iii) statistical analysis of data; (iv) low-pass filtering and decimation. In [10-11], the modal parameters of the tower were extracted from the measured acceleration data using an automated procedure [2] based on the covariance-driven Stochastic Subspace Identification (SSI-Cov) algorithm. In the present paper, another procedure (still based on the SSI-Cov method and extensively described in another paper submitted in the VETOMAC conference) was used for automated OMA.

As described in [10-11], five vibration modes were identified in the preliminary ambient vibration tests and the corresponding natural frequencies were successfully tracked during the monitoring period. The identified modes correspond to three bending modes, one torsion mode and one local mode involving the upper part of the structure. For additional details on the vibration modes of the tower (identified in ambient vibration tests and tracked during the structural monitoring), the interested reader is referred to [10-11]. 
It should be noticed that, until June 2013, the tower's response to different far-field earthquakes was recorded by the monitoring system. The strongest event - corresponding to an earthquake occurred in the Garfagnana region (Tuscany) on June 21st, 2013 - was characterized by a measured peak acceleration of about $20 \mathrm{~cm} / \mathrm{s}^{2}$, exceeding about 50 times the highest amplitude of normally observed ambient vibrations.

a)

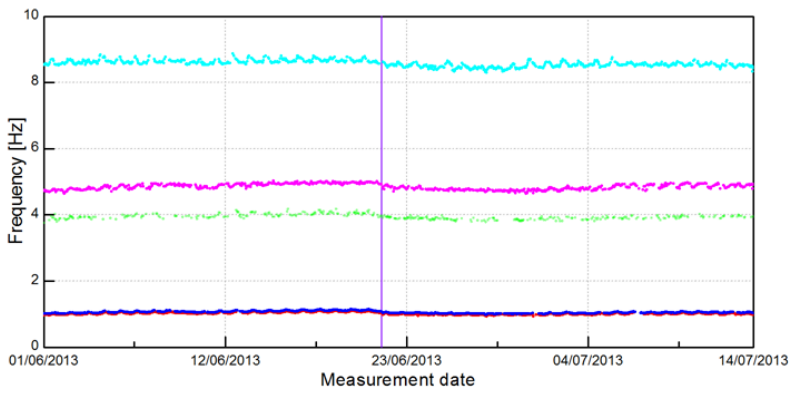

b)

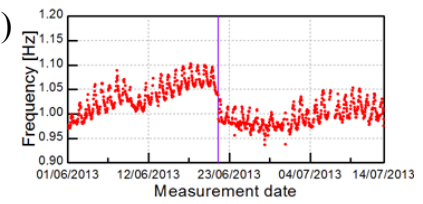

c)

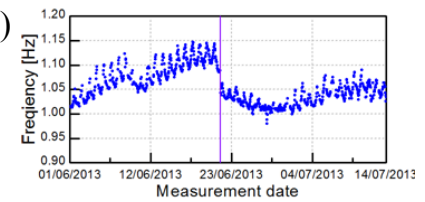

Fig. 2. a) Evolution in time of natural frequencies obtained from $01 / 06 / 2013$ to $13 / 07 / 2013$. Zoom of the frequency evolution associated to b) the first mode and c) the second mode.

The time evolution of the automatically identified natural frequencies, from 01/06/2013 to 13/07/2013, is shown in Fig. 2. The good performance of the OMA procedure is firstly demonstrated by the excellent correspondence with the previously obtained results [10-11]. In addition, Fig. 2 generally shows high values of the identification success rate (SR) for each mode. More specifically, during the monitoring period (43 days, 1032 1-hour datasets) investigated in Fig. 2, the natural frequency of modes 1-2 and 4-5 was identified with SR ranging between $92.4 \%$ (mode 1 ) and $83.7 \%$ (mode 4 ), whereas the $\mathrm{SR}=36,5 \%$ for mode 3 . As already noticed in [11], the lower SR for mode 3 is due to the presence of spurious harmonics close to the natural frequency of this mode.

According to the tracking results, only the frequency data characterized by the higher SR values (i.e., modes 1-2 and 4-5) were used as input for the SVM algorithm.

\subsection{Detecting the structural changes through the SVM-based strategy}

As previously stated - on June 21st, 2013 - the tower was subjected to a seismic event which led to slight structural damage (see Fig. 2). This damage was not observed by visual inspection but it was clearly detected through permanent frequency shifts (decreases of $2 \%$, $1 \%$ and $0.5 \%$ for the first two bending modes and the torsion mode of the tower) as well as by applying various SHM strategies [10-11]. The application of the SVM also provides evidence of the abnormal change, using selected intervals of data in which the SVM model is forced to recognize two difference scenarios, associating the $50 \%$ of input data to undamaged state and $50 \%$ to damage state, as shown Fig. 3.
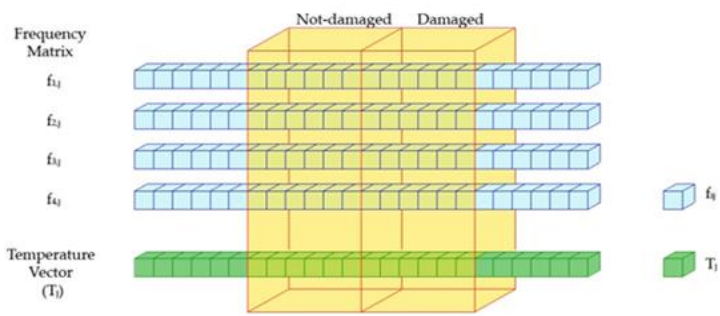

Fig. 3. Input data for the SVM procedure: natural frequencies matrix and temperature vector. 
(a) $1^{\text {st }}$ modal frequency

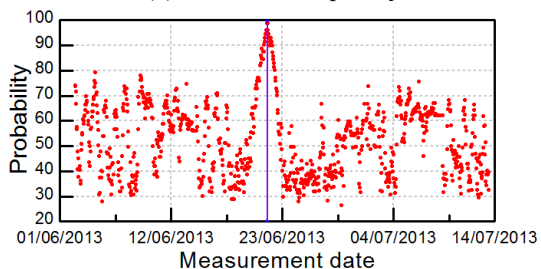

(b) $2^{\text {nd }}$ modal frequency

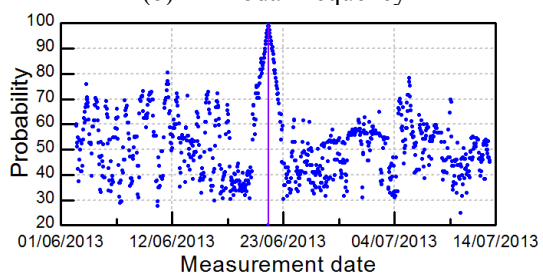

(c) $4^{\text {th }}$ modal frequency

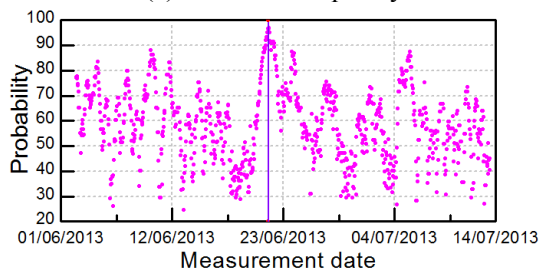

(d) $5^{\text {th }}$ modal frequency

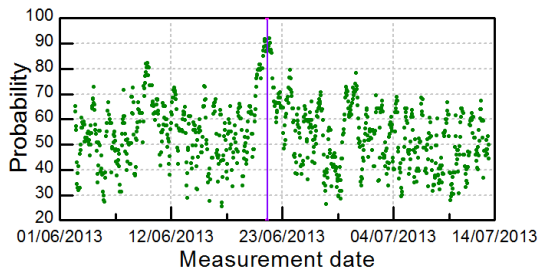

(e) $1^{\text {st }}$ modal frequency + measured temperature

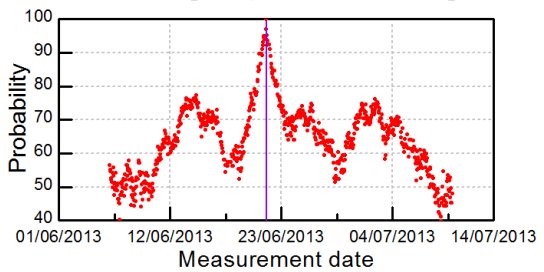

(f) $2^{\text {nd }}$ modal frequency + measured temperature

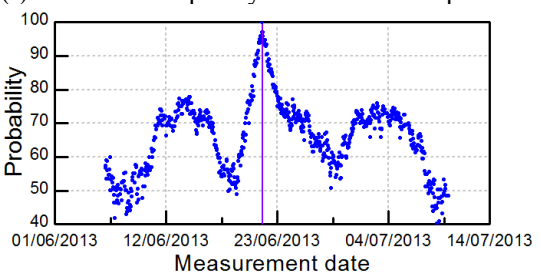

(g) $4^{\text {th }}$ modal frequency + measured temperature

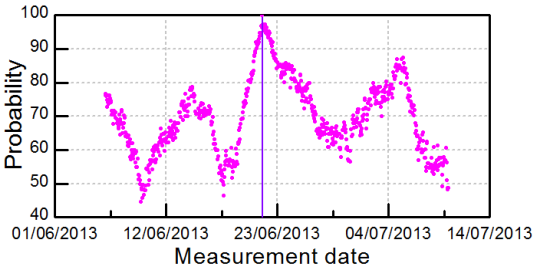

(h) $5^{\text {th }}$ modal frequency + measured temperature

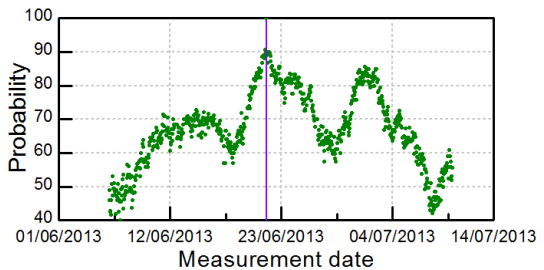

Fig. 4. Damage prediction provided by the classification of each data series of identified frequency: (a)-(d) results obtained by using only the $i$-th modal frequency as input of the SVM; (e)-(h) results obtained by using the $i$-th modal frequency and the measured temperature as inputs of the SVM.

(a) modal frequencies

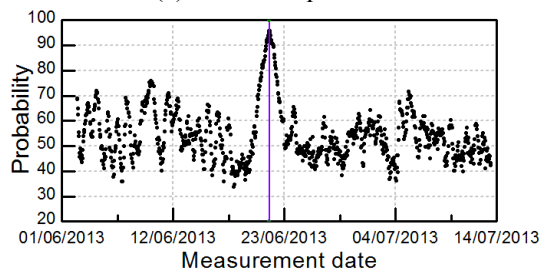

(b) modal frequencies + measured temperature

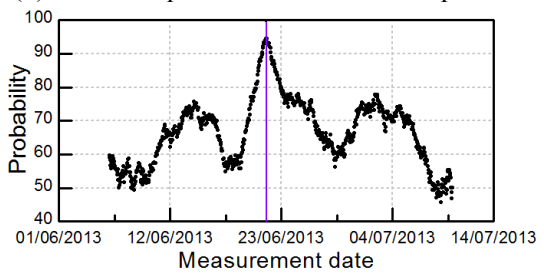

Fig. 5. Average damage prediction probability: a) First strategy, 2) Second strategy

Firstly, the SVM was used to classify each series of frequency data that were organized in intervals of 96 hours. The SVM probability is an indicator of the occurrence of an abnormal behaviour, with higher peak value being associated to better data classification. When the probability reaches $100 \%$, it means that all data were perfectly associated to different classes (i.e., undamaged and damaged scenario) and this condition is only possible if the change (i.e., the damage) is located exactly in the middle of the interval of input data. 
Results obtained by the application of this first strategy are shown in Figs. 4a-d, with the clearest novelty indications being associated to the first two frequency evolutions (Figs. 4a and $4 \mathrm{~b}$ ). It is worth noting that, even if no information is provided on the changing environment, the absolute maximum of the probability of all modes in Figs. 4a-d exactly corresponds to the occurrence of the seismic event.

In order to better investigate the effects of the environmental conditions (i.e. temperature) that could provide possible misclassification, both the natural frequencies and the measured temperature were adopted as input of the SVM model [12]. The analysis is basically carried out in a similar way to the previous one and again the SVM probability peaks, for all modes, when the earthquake occurred (Figs. 4e-h). The main difference between the two SVM applications (beyond the use of different inputs) is the dimension of the adopted time intervals, that were increased to 240 hours (120 hours for each state, see Fig. 3). Hence, it seems that the unsupervised approach works better (or simply with lower amount of data) if the classification does not involve also the temperature, probably as a consequence of the high variability of the temperature in the investigated period.

Finally, in order to make more legible the obtained results, the predictions of the SVM model for each natural frequency of both approaches were put together and average results for both strategies are shown in Fig. 5. Again, the inspection of Fig. 5 highlights that the main peaks of the average SVM probability correspond to the damaging earthquake.

The presented results show an interesting promise about the possibility of performing a quite simple OMA-based SHM of historic towers, even using low-cost monitoring systems.

\section{Conclusions}

This paper focuses on a SHM strategy based on SVM classification of the frequency data obtained by automated OMA. In order to validate the proposed methodology, experimental data collected in the continuous dynamic monitoring of an ancient tower were used. During the monitoring, the tower underwent slight permanent variation of the natural frequencies after the occurrence of a far-field earthquake.

The SVM classification was carried out using only the automatically identified frequency data and coupling these data with temperature measurements. In both cases, the SVM algorithm demonstrated its capability of clearly highlighting the occurrence of structural changes exactly corresponding to the seismic event.

\section{References}

1. F. Magalhães, Á. Cunha, E. Caetano, Mech. Syst. Signal Pr. 23, 316-329, (2009)

2. A. Cabboi, F. Magalhães, C. Gentile, Á. Cunha, Struct. Control Hlth 24(1), (2017)

3. E. Reynders, J. Houbrechts, G. De Roeck, Mech. Syst. Signal Pr. 29, 228-250, (2012)

4. R. Cardoso, A. Cury, F. Barbosa, Mech. Syst. Signal Pr. 95, 24-41, (2017)

5. H. Sohn, Phil. Trans. R. Soc. A 365, 539-560 (2007)

6. B. Peeters, G. De Roeck, Earthq. Eng. Struct. D. 30, 149-171, (2001)

7. F. Magalhães, Á. Cunha, E. Caetano, Mech. Syst. Signal Pr. 28, 212.228, (2012)

8. C. K. Oh, H. Sohn, J Sound Vib. 1-2, 224-239, (2009)

9. H. Sohn, K. Worden, C.R. Farrar CR, J. Intell. Mater. Syst. Struct. 13, 561-574 (2002)

10. A. Saisi, C. Gentile, M. Guidobaldi, Constr. Build. Mater. 81, 101-112, (2015)

11. C. Gentile, M. Guidobaldi, A. Saisi, Meccanica 51, 2873-2889, (2016)

12. R. P. Finotti, F. Barbosa, A. Cury, C. Gentile, Procedia Engineering 199, (2017) 